\title{
FLOW OF A VISCO-ELASTIC FLUID PAST A POROUS PLATE IN A ROTATING SYSTEM
}

\author{
M. GURIA* \\ Department of Mathematics \\ Ghatal Rabindra Satabarshiki Mahavidyalaya \\ Ghatal, Paschim Medinipur, West Bengal, INDIA \\ E-mail: mrinmoy9832@yahoo.com \\ R.N. JANA \\ Department of Applied Mathematics \\ with Oceanology and Computer Programming \\ Vidyasagar University, Midnapore-721102 \\ West Bengal, INDIA
}

\begin{abstract}
An analysis is made on a steady flow of an incompressible visco-elastic fluid (of small memory) past a porous plate subject to uniform suction or blowing. The fluid and the plate rotate in unison with uniform angular velocity about an axis perpendicular to the plate. It is shown that for a visco-elastic fluid of small memory, a boundary layer is formed for uniform suction or blowing at the plate. It is found that the primary velocity increases whereas the secondary velocity increases near the plate and decreases away from the plate with an increase in the elastic parameter. The secondary flow shows flow reversal for a large elastic parameter. It is found that both primary and secondary velocities increase near the plate and decrease away from the plate with an increase in the rotation parameter. It is also found that the shear stress due to primary flow increases with an increase in either the elastic parameter or rotation parameter. On the other hand, the shear stress due to secondary flow decreases with an increase in the elastic parameter while it increases with an increase in the rotation parameter. The temperature distribution in the boundary layer is also determined. It is shown that elasticity leads to an increase in heat transfer at the plate.
\end{abstract}

Key words: visco-elastic, relaxation, back flow, elasticity.

\section{Introduction}

It is known that liquids respond like elastic solids to impulses, which are very rapid compared to the time, it takes for the molecular order associated with short-range forces in the liquid to relax. For liquids with small molecules this time of relaxation is estimated as $10^{-13}$ or $10^{-10}$ seconds depending on the fluids. Waves associated with such liquids move with speeds of $10^{5} \mathrm{~cm} / \mathrm{s}$ or even faster (Joseph, 1990). There also exist liquids which have much longer times of relaxation. Polymers mixed in Newtonian solvents, polymer melts like molten plastics or high viscosity silicone oils are examples. These types of fluids are known as viscoelastic fluids. The longest times of relaxation for these fluids are of practical interest. Such fluids have become important industrially, particularly in polymer processing applications as well as in chemical industries, one deals with flow of visco-elastic fluids. The fluctuating flow of a visco-elastic fluid past an infinite porous plate subject to uniform suction was studied by Kaloni (1966). The steady flow of an incompressible second order fluid past an infinite porous plate subject to suction or blowing was investigated by Rajagopal and Gupta (1984).

\footnotetext{
* To whom correspondence should be addressed
} 
In this paper, we investigate momentum and heat transfer in the steady flow of a visco-elastic fluid (obeying Walter's liquid B' model (1964)) past an infinite porous flat plate in a rotating system subject to uniform suction or blowing.

\section{Formulation of the problem}

Consider a steady flow of an incompressible visco-elastic fluid past an infinite porous flat plate subject to uniform suction. The fluid and the plate rotate in unison with a constant angular velocity $\Omega$ about an axis perpendicular to the plate [Fig.1].

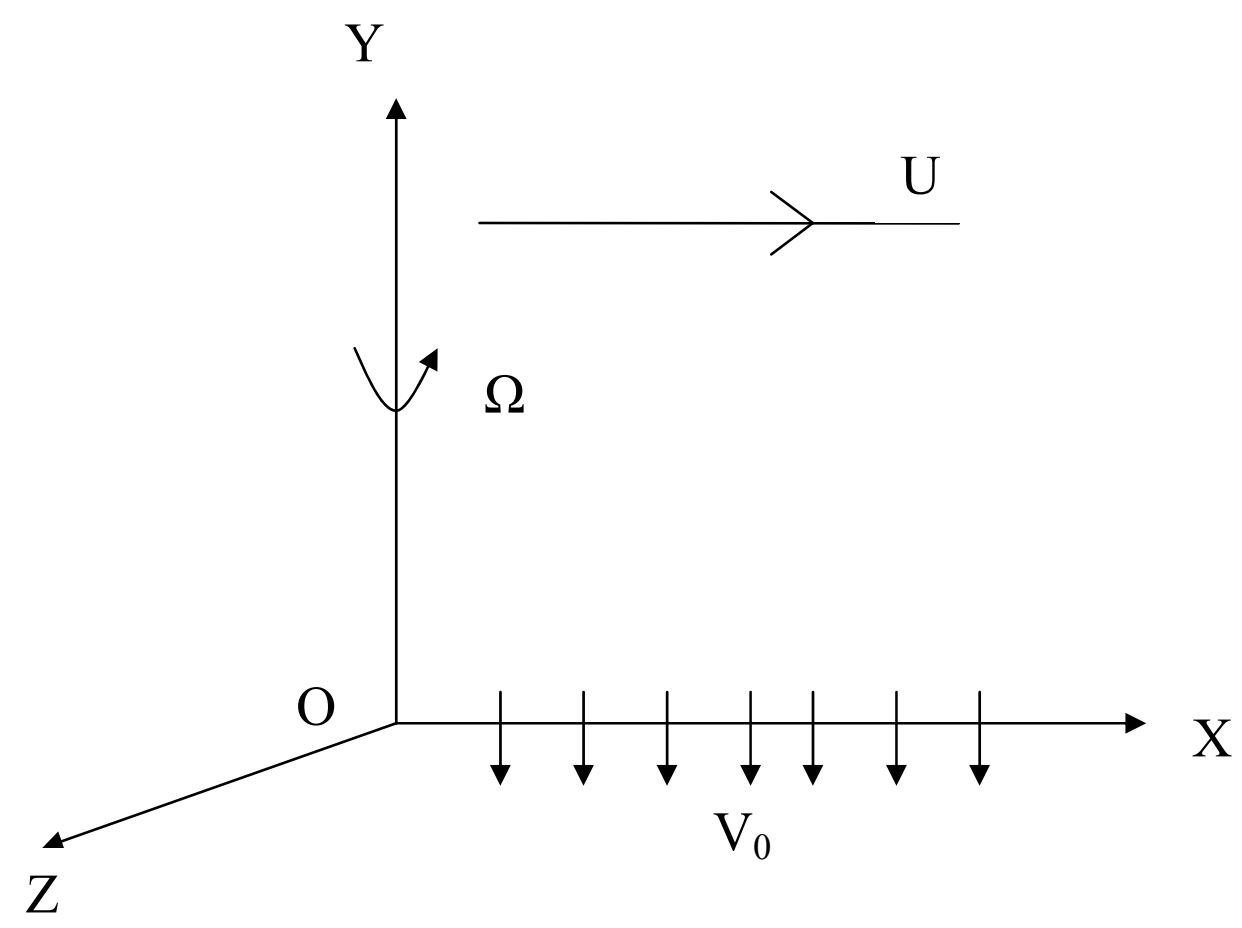

Fig.1. Geometry of the problem.

The constitutive equations characterizing the visco-elastic fluid (obeying Walter's liquid $B^{\prime}$ model (1964)) are

$$
\begin{aligned}
& p_{i k}=-p g_{i k}+p_{i k}^{\prime}, \\
& p_{i k}^{\prime}=2 \int_{-\infty}^{t} \psi\left(t-t^{\prime}\right) \frac{\partial x^{\prime}}{\partial x^{\prime m}} \frac{\partial x^{k}}{\partial x^{\prime r}} e^{(I) m r} d t^{\prime},
\end{aligned}
$$

where $p_{i k}$ is the stress tensor, $p$ is an arbitrary isotropic pressure, $g_{i k}$ is the metric tensor, $e^{(1) m k}$ is the rate of strain tensor and

$$
\psi\left(t-t^{\prime}\right)=\int_{0}^{\infty} \frac{N(\tau)}{\tau} \exp \left[-\left(t-t^{\prime}\right) / \tau\right] d \tau
$$


where $N(\tau)$ is the distribution function of the relaxation time $\tau$. Beard and Walters (196) showed that in the case of liquids with short memories (i.e., a short relaxation time) the above equation gives

$$
p_{i k}^{\prime}=2 \eta_{0} e^{(1) i k}-2 k_{0} \frac{\delta e^{(1) i k}}{\delta t}
$$

where $\eta_{0}=\int_{0}^{\infty} N(\tau) d \tau$ is the limiting viscosity at small rate of shear, $k_{0}=\int_{0}^{\infty} \tau N(\tau) d \tau$ is the short memory coefficient and $\frac{\delta}{\delta t}$ denotes the convected differentiation of a tensor which for any contravarient tensor is given by

$$
\frac{\delta b^{i k}}{\delta t}=\frac{\partial b^{i k}}{\partial t}+v^{m} \frac{\partial b^{i k}}{\partial x^{m}}-v^{i m} \frac{\partial v^{i k}}{\partial x^{m}}-b^{m k} \frac{\partial v^{i}}{\partial x^{m}}
$$

where $v^{i}$ is the velocity vector.

We take the $x$ - axis along the direction of the flow, the $z$-axis is perpendicular to the $x y$-plane and the $y$ - axis is normal to it [Fig.1]. Since the plate is infinite, in the steady state all the physical variables except pressure depend on $y$ only.

We assume the velocity field of the form

$$
v^{(1)}=u(y), \quad v^{(2)}=v(y), \quad v^{(3)}=w(y),
$$

The equation of continuity $\nabla \cdot \boldsymbol{q}=0$ gives

$$
v=-v_{0}
$$

where $v_{0}$ is the constant velocity at the plate, $v_{0}>0$ for suction and $v_{0}<0$ for blowing.

The equations of momentum for a visco-elastic fluid, in a rotating frame of reference along the $x-, y-$ and $z-$ axes are given by

$$
\begin{aligned}
& -v_{0} \frac{d u}{d y}-2 \Omega w=-\frac{1}{\rho} \frac{\partial p}{\partial x}+\frac{k_{0} v_{0}}{\rho} \frac{d^{3} u}{d y^{3}}+\mathrm{v} \frac{d^{2} u}{d y^{2}}, \\
& 0=-\frac{1}{\rho} \frac{\partial p}{\partial y}, \\
& -v_{0} \frac{d w}{d y}+2 \Omega u=-\frac{1}{\rho} \frac{\partial p}{\partial z}+\frac{k_{0} v_{0}}{\rho} \frac{d^{3} w}{d y^{3}}+v \frac{d^{2} w}{d y^{2}},
\end{aligned}
$$

where $p$ is the pressure, $\rho$ is the density of the fluid. $v$ is the kinematic coefficient of viscosity.

The boundary conditions are 


$$
u=w=0 \quad \text { at } \quad y=0, \quad u \rightarrow U, \quad w \rightarrow 0 \quad \text { as } \quad y \rightarrow \infty .
$$

Using infinity conditions, Eqs (2.8) and (2.10) become

$$
\begin{aligned}
& -v_{0} \frac{d u}{d y}-2 \Omega w=v \frac{d^{2} u}{d y^{2}}+\frac{k_{0} v_{0}}{\rho} \frac{d^{3} u}{d y^{3}} \\
& -v_{0} \frac{d w}{d y}+2 \Omega(u-U)=v \frac{d^{2} w}{d y^{2}}+\frac{k_{0} v_{0}}{\rho} \frac{d^{3} w}{d y^{3}} .
\end{aligned}
$$

Introducing, the non-dimensional variables

$$
\eta=\frac{U y}{v}, \quad u_{1}=\frac{u}{U}, \quad w_{1}=\frac{w}{U},
$$

equations (2.12) and (2.13) become

$$
\begin{aligned}
& \frac{d^{2} u_{1}}{d \eta^{2}}+\sigma S \frac{d^{3} u_{1}}{d \eta^{3}}+S \frac{d u_{1}}{d \eta}+2 K^{2} w_{1}=0 \\
& \frac{d^{2} w_{1}}{d \eta^{2}}+\sigma S \frac{d^{3} w_{1}}{d \eta^{3}}+S \frac{d w_{1}}{d \eta}-2 K^{2}\left(u_{1}-1\right)=0,
\end{aligned}
$$

where $S=v_{0} / U$ is the suction parameter, $\sigma=k_{0} U^{2} / \rho v^{2}$ is the visco-elastic parameter, and $K^{2}=2 \Omega v / U^{2}$ is the rotation parameter.

The boundary conditions (2.11) become

$$
u_{1}=w_{1}=0 \quad \text { at } \quad \eta=0, \quad u_{1}=1, \quad w_{1}=0 \quad \text { as } \quad \eta \rightarrow \infty .
$$

Following, Ray Mahapatra and Gupta (2004), we seek a solution of the form

$$
\begin{aligned}
& u_{1}=u_{10}(\eta)+\sigma u_{11}(\eta)+\sigma^{2} u_{12}(\eta)+\ldots \\
& w_{1}=w_{10}(\eta)+\sigma w_{11}(\eta)+\sigma^{2} w_{12}(\eta)+\ldots
\end{aligned}
$$

which are valid for a sufficiently small $\sigma$. Substituting Eqs (2.18) in Eqs (2.15) and (2.16) and equating the term independent of $\sigma$ and coefficient of $\sigma$, we get

$$
\begin{aligned}
& \frac{d^{2} u_{10}}{d \eta^{2}}+S \frac{d u_{10}}{d \eta}+2 K^{2} w_{10}=0, \\
& \frac{d^{2} w_{10}}{d \eta^{2}}+S \frac{d w_{10}}{d \eta}-2 K^{2}\left(u_{10}-1\right)=0,
\end{aligned}
$$




$$
\begin{aligned}
& \frac{d^{2} u_{11}}{d \eta^{2}}+S \frac{d u_{11}}{d \eta}+2 K^{2} w_{11}=-S \frac{d^{3} u_{10}}{d \eta^{3}} \\
& \frac{d^{2} w_{11}}{d \eta^{2}}+S \frac{d w_{11}}{d \eta}-2 K^{2} u_{11}=-S \frac{d^{3} w_{10}}{d \eta^{3}}
\end{aligned}
$$

The corresponding boundary conditions are

$$
u_{10}=w_{10}=u_{11}=w_{11}=0 \text { at } \eta=0 \text { and } u_{10}=1, w_{10}=0, u_{11}=w_{11}=0 \text { as } \eta \rightarrow \infty .
$$

The solutions of Eqs (2.19)-(2.22), subject to the boundary conditions (2.23) are

$$
\begin{aligned}
& u_{10}(\eta)=1-e^{-(S / 2+\alpha) \eta} \cos \beta \eta, \\
& w_{10}(\eta)=e^{-(S / 2+\alpha) \eta} \sin \beta \eta \\
& u_{11}(\eta)=\left(a_{1} \cos \beta \eta+b_{1} \sin \beta \eta\right) \eta e^{-(S / 2+\alpha) \eta}, \\
& w_{11}(\eta)=\left(b_{1} \cos \beta \eta-a_{1} \sin \beta \eta\right) \eta e^{-(S / 2+\alpha) \eta}
\end{aligned}
$$

where

$$
\begin{aligned}
& \alpha, \beta=\frac{1}{2 \sqrt{2}}\left[\left(S^{4}+64 K^{4}\right)^{1 / 2} \pm S^{2}\right]^{1 / 2}, \\
& a_{1}=\frac{S}{\left(\alpha^{2}+\beta^{2}\right)}\left[\alpha\left\{\left(\frac{S}{2}+\alpha\right)^{3}-3\left(\frac{S}{2}+\alpha\right) \beta^{2}\right\}+\beta\left\{3\left(\frac{S}{2}+\alpha\right)^{2} \beta-\beta^{3}\right\}\right] \\
& b_{1}=\frac{S}{\left(\alpha^{2}+\beta^{2}\right)}\left[\alpha\left\{3\left(\frac{S}{2}+\alpha\right)^{2} \beta-\beta^{2}\right\}-\beta\left\{\left(\frac{S}{2}+\alpha\right)^{3}-3\left(\frac{S}{2}+\alpha\right) \beta^{2}\right\}\right] .
\end{aligned}
$$

Hence the velocity distributions, up to order $O(\sigma)$, are

$$
\begin{aligned}
& u_{l}(\eta)=1-\left[\cos \beta \eta-\sigma\left(a_{1} \cos \beta \eta+b_{1} \sin \beta \eta\right) \eta\right] e^{-(S / 2+\alpha) \eta} \\
& w_{l}(\eta)=\left[\sin \beta \eta+\sigma\left(b_{1} \cos \beta \eta-a_{1} \sin \beta \eta\right) \eta\right] e^{-\left(S / 2^{+\alpha}\right) \eta}
\end{aligned}
$$

In the case of blowing at the plate $(S<0)$ an asymptotic solution is also possible and the solution for blowing is obtained by letting $S=-S_{1}$ with $\left(S_{1}>0\right)$ in Eqs (2.28)-(2.30).

In the absence of rotation $\left(K^{2}=0\right)$, the above Eqs (2.29) and (2.30) become 


$$
u_{1}(\eta)=1-e^{-S \eta}+2 S^{3} \sigma \eta e^{-S \eta} \quad \text { and } \quad w_{1}(\eta)=0 .
$$

In this case no steady distributions exist for uniform blowing at the plate.

\section{Results and discussion}

To study flow situations for different values of the elastic parameter $\sigma$, rotation parameter $K^{2}$ and the suction parameter $S$, the velocity profiles are examined numerically and plotted in Figs 2-4. Figure 2 shows the variations of the primary and the secondary velocity profiles for different values of the elastic parameter $\sigma$ for $K^{2}=4.0$ and $S=1.0$. It is found that the primary velocity increases whereas the secondary velocity increases near the plate and decreases away from the plate with an increase in the elastic parameter $\sigma$. The secondary flow shows flow reversal for a large elastic parameter. The variation of velocity profiles for different values of the rotation parameter $K^{2}$ is shown in Fig. 3 for $\sigma=0.01, S=1.0$. It is evident from Fig. 3 that both the primary and the secondary velocities increase near the plate and decrease away from the plate with an increase in the rotation parameter $K^{2}$ while a back flow appears in the secondary flow for high rotation. Thus rotation exerts a stabilizing influence on the primary and secondary flows near the plate. Fig. 4 shows the velocity distributions $u_{1}$ and $w_{1}$ for several values of $S$ with $\sigma=0.01, K^{2}=4.0$. It is observed that the primary velocity increases near the plate and decreases away from the plate with an increase in the suction parameter $S$. On the other hand, the secondary flow decreases with an increase in the suction parameter $S$ while a back flow occurs for large suction.

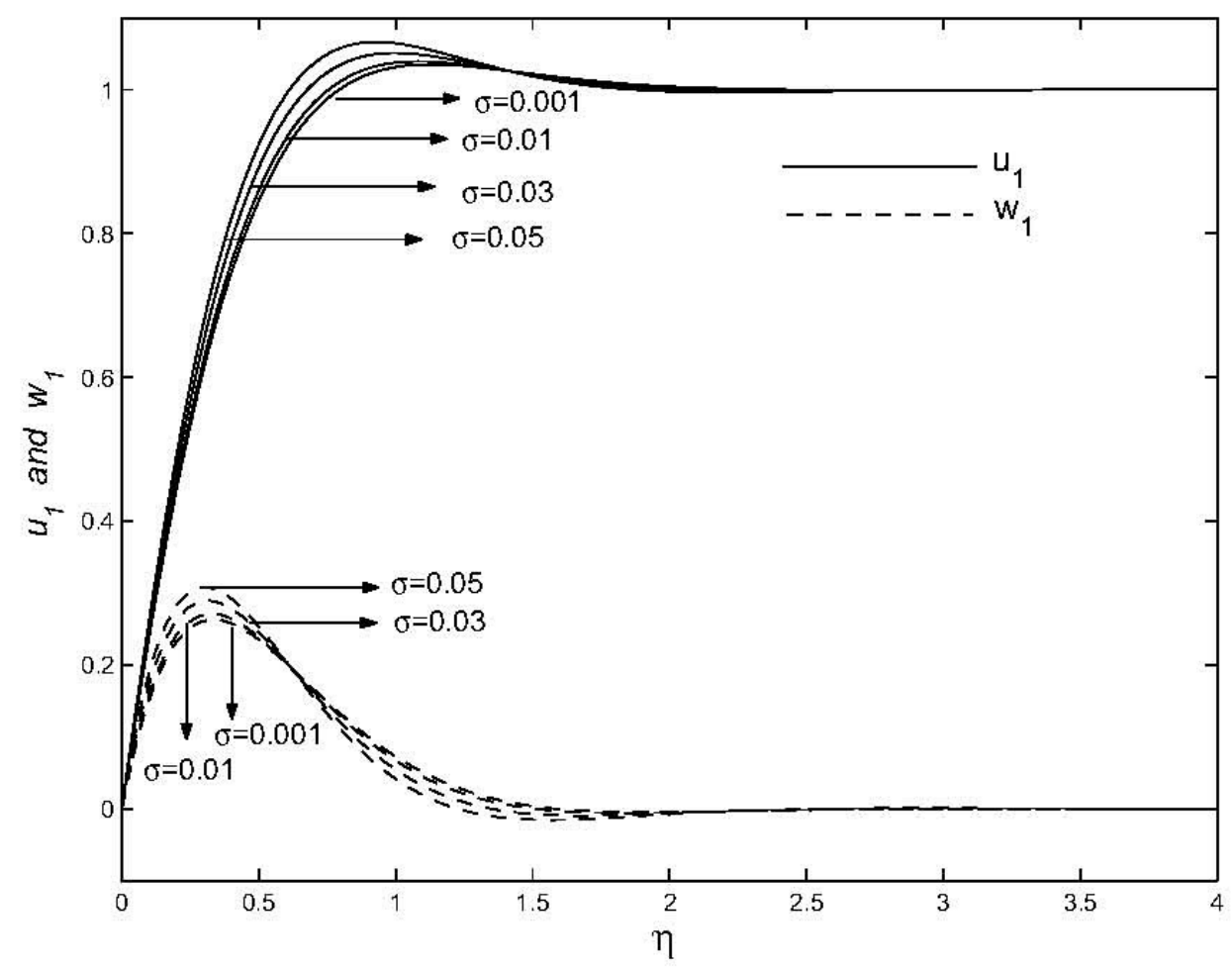

Fig.2. Variations of velocity profiles $u_{1}$ and $w_{1} K_{2}=4.0, S=1.0$. 


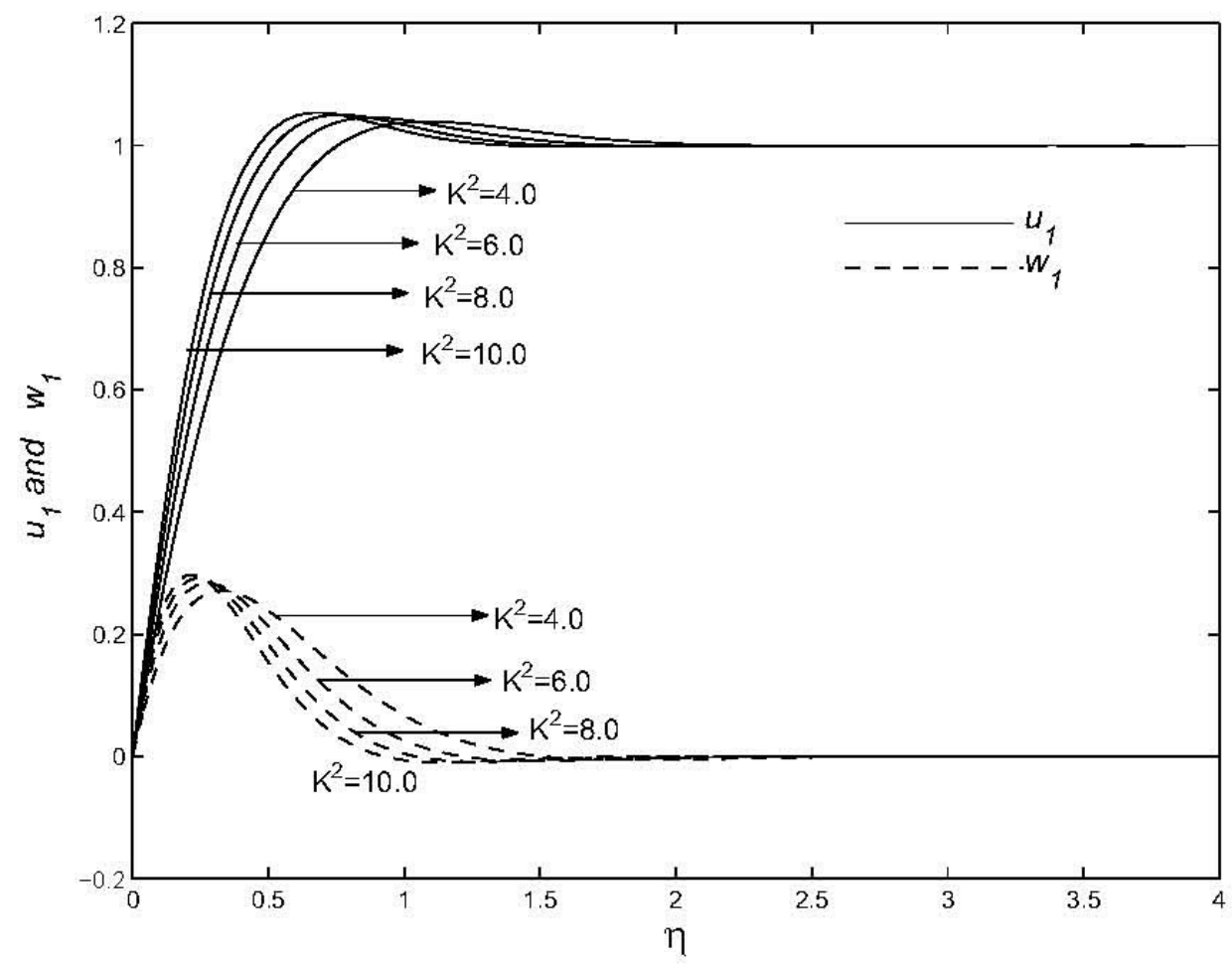

Fig.3. Variations of velocity profiles $u_{1}$ and $w_{1} \sigma=0.01, S=1.0$

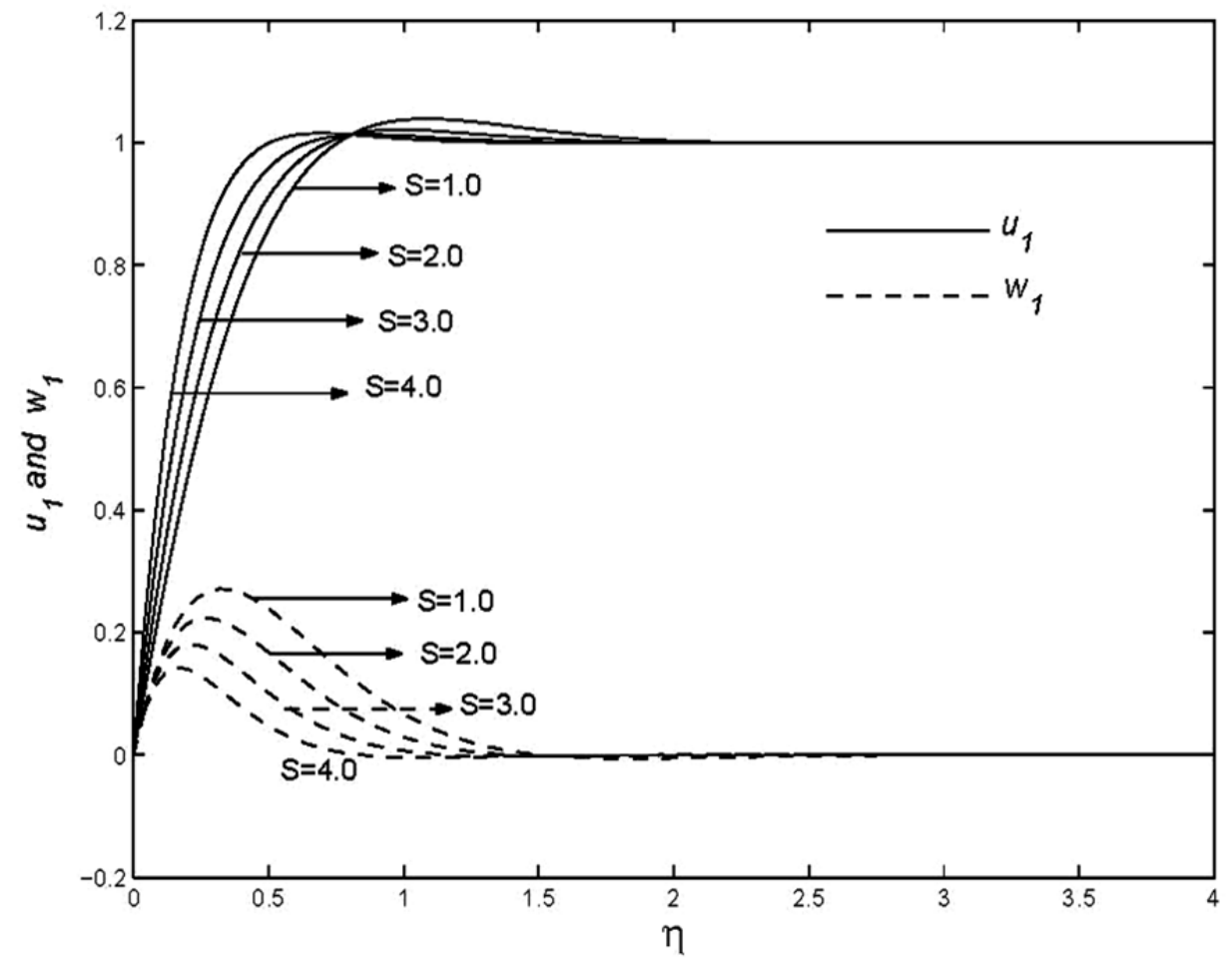

Fig.4. Variations of velocity profiles $u_{1}$ and $w_{1} \sigma=0.01, K^{2}=4.0$. 
The velocity profiles $u_{l}$ and $w_{l}$ are plotted in Fig.5 for different values of the blowing parameter with $\sigma=0.01, K^{2}=4.0$. It is seen that the primary velocity decreases near the plate and increases away from the plate and then decreases and ultimately reaches a steady state. On the other hand, $w_{l}$ increases near the plate and decreases away from the plate with an increase in the blowing parameter.

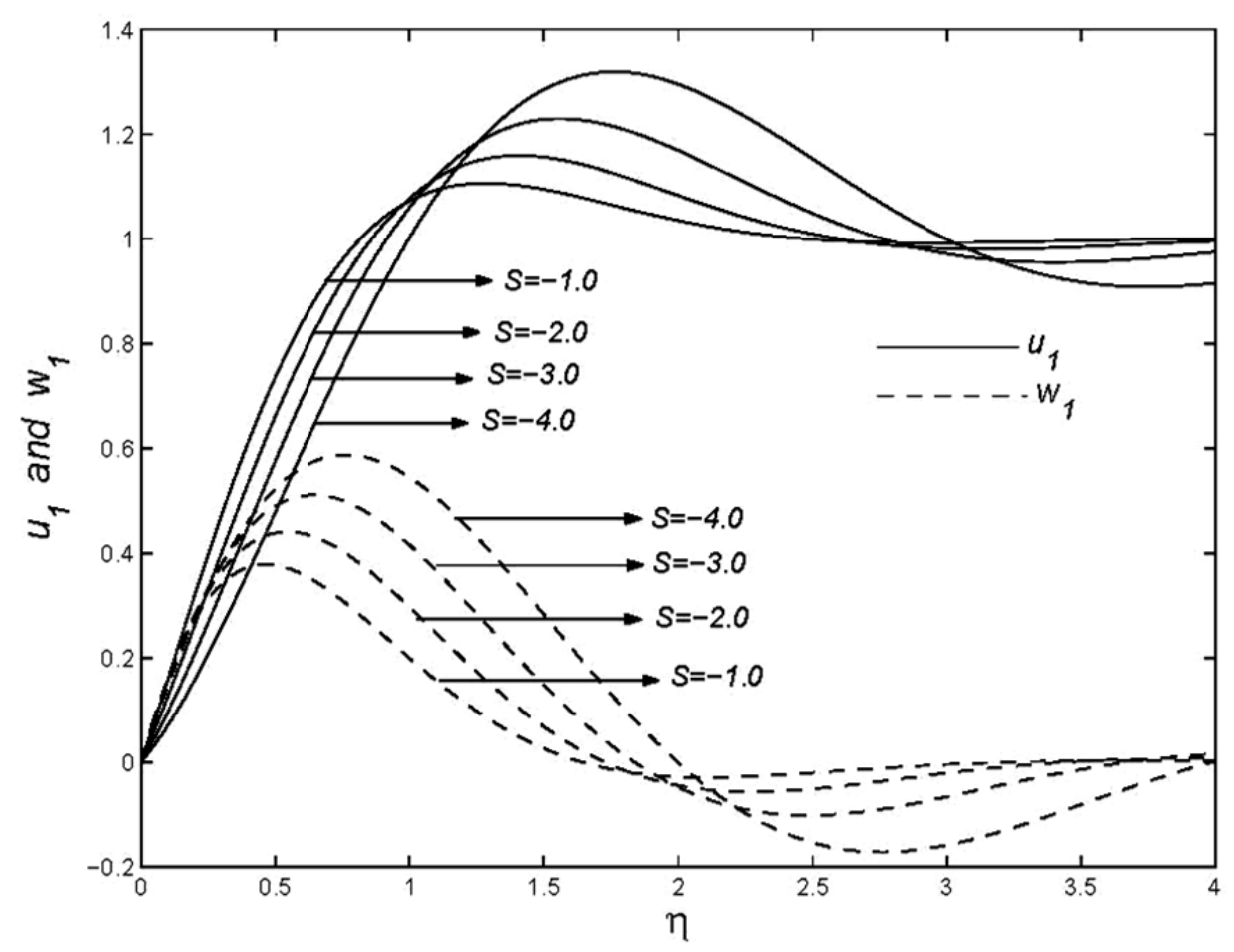

Fig.5. Variations of velocity profiles $u_{1}$ and $w_{1} \sigma=0.01, K^{2}=4.0$.

The shear stresses due to the primary and the secondary velocities at the plate $y=0$ are given by

$$
\begin{gathered}
\tau_{x}^{*}=\left(\mu \frac{d u}{d y}+k_{0} v_{0} \frac{d^{2} u}{d y^{2}}\right)_{y=0}, \\
\tau_{y}^{*}=\left(\mu \frac{d w}{d y}+k_{0} v_{0} \frac{d^{2} w}{d y^{2}}\right)_{y=0},
\end{gathered}
$$

In a non-dimensional form the shear stress due to primary and secondary flows at the plate $\eta=0$ can be obtained as

$$
\begin{aligned}
& \tau_{x}=\frac{\tau_{x}^{*} \mathrm{v}}{\mu U^{2}}=\left(\frac{S}{2}+\alpha\right)-\sigma S\left[\left(\frac{S}{2}+\alpha\right)^{2}-\beta^{2}\right]+\sigma a_{1}-2 \sigma^{2} S\left\{a_{1}\left(\frac{S}{2}+\alpha\right)-\beta b_{1}\right\}, \\
& \tau_{y}=\frac{\tau_{y}^{*} \mathrm{v}}{\mu U^{2}}=\beta-2 \sigma S \beta\left(\frac{S}{2}+\alpha\right)+\sigma b_{1}-2 \sigma^{2} S\left\{b_{1}\left(\frac{S}{2}+\alpha\right)+a_{l} \beta\right\} .
\end{aligned}
$$


The non-dimensional shear stress components $\tau_{x}$ and $\tau_{y}$ are given in Tabs 1 and 2 for different values of the rotation parameter $K^{2}$ and elastic parameter $\sigma$. It is seen that at a fixed instant both $\tau_{x}$ and $\tau_{y}$ increase with an increase in the rotation parameter $K^{2}$. These results have a physical interpretation. At a fixed instant, an increase in $K^{2}$ causes a gradual thinning of the boundary layer which develops on the plate.

Table.1. Shear stress due to primary flow for $S=1.0$.

\begin{tabular}{|l|cccc|}
\hline & \multicolumn{3}{|c|}{$\tau_{x}$} \\
\hline$K^{2}-10.01$ & 0.03 & 0.05 \\
\hline 4.0 & 0.001 & 0.01 & 2.66476 \\
6.0 & 2.53306 & 2.54920 & 2.59804 & 3.18202 \\
8.0 & 2.97694 & 2.99751 & 3.07077 & 3.64250 \\
10.0 & 3.35263 & 3.37786 & 3.47906 & 4.06901 \\
\hline
\end{tabular}

On the other hand, for a fixed value of $K^{2}, \tau_{x}$ increases whereas $\tau_{y}$ decreases with an increase in $\sigma$. This shows that the wall shear stress due to secondary flow decreases with an increase in elasticity of the fluid.

Table.2. Shear stress due to secondary flow for $S=1.0$.

\begin{tabular}{|c|c|c|c|c|}
\hline & \multicolumn{4}{|c|}{$\tau_{y}$} \\
\hline$K^{2}>\sigma$ & 0.001 & 0.01 & 0.03 & 0.05 \\
\hline 4.0 & 1.96988 & 1.96139 & 1.93338 & 1.83827 \\
\hline 6.0 & 2.42517 & 2.42439 & 2.35543 & 2.19379 \\
\hline 8.0 & 2.80763 & 2.80372 & 2.69933 & 2.46293 \\
\hline 10.0 & 3.14390 & 3.13626 & 2.99268 & 2.67449 \\
\hline
\end{tabular}

\section{Temperature distribution}

Consider the heat transfer equation in the flow of a visco-elastic fluid. To this end it is necessary to establish the energy balance for a fluid element in motion and to consider it in conjunction with the equation of motion. It is to be noted that during the motion of a visco-elastic fluid, a certain amount of energy is stored up in the fluid as strain energy and some energy is lost due to viscous dissipation. Thus for such a fluid, the energy balance is determined by the internal energy, the conduction of heat, the convection of heat with the flow, the generation of heat through viscous dissipation and the strain (or deformation) energy stored in the fluid due to its elastic properties.

The transfer of heat in the steady flow of visco-elastic fluid past a flat porous plate can be expressed in the form of the energy equation given by 


$$
-v_{0} \frac{d T}{d y}=\frac{\lambda}{\rho C_{p}} \frac{d^{2} T}{d y^{2}}+\frac{\mu}{\rho C_{p}}\left[\left(\frac{d u}{d y}\right)^{2}+\left(\frac{d w}{d y}\right)^{2}\right]+\frac{k_{0} v_{0}}{\rho C_{p}}\left[\frac{d u}{d y} \frac{d^{2} u}{d y^{2}}+\frac{d w}{d y} \frac{d^{2} w}{d y^{2}}\right],
$$

where $T, \lambda$ and $C_{p}$ denote the temperature, the thermal diffusivity and the specific heat of the fluid, respectively. The second and third terms on the right hand side of Eq.(4.1) are due to viscous dissipation and strain energy, respectively.

The boundary conditions are

$$
T=T_{w} \quad \text { at } \quad y=0, \quad T \rightarrow T_{\infty} \quad \text { as } \quad y \rightarrow \infty
$$

where $T_{w}$ and $T_{\infty}\left(T_{w}>T_{\infty}\right)$ are the temperature at the plate and at infinity, respectively. Introducing the dimensionless temperature

$$
\theta=\frac{T-T_{\infty}}{T_{w}-T_{\infty}}
$$

and on using Eq.(2.9), Eq.(4.1) becomes

$$
\frac{d^{2} \theta}{d \eta^{2}}+S \operatorname{Pr} \frac{d \theta}{d \eta}=-P_{r} E_{c}\left[\left(\frac{d u_{1}}{d \eta}\right)^{2}+\left(\frac{d w_{1}}{d \eta}\right)^{2}+\sigma S\left\{\frac{d u_{1}}{d \eta} \cdot \frac{d^{2} u_{1}}{d \eta^{2}}+\frac{d w_{1}}{d \eta} \cdot \frac{d^{2} w_{1}}{d \eta^{2}}\right\}\right]
$$

where $\operatorname{Pr}=\frac{\mu C_{p}}{\lambda}$ is the Prandtl number and $\mathrm{Ec}=\frac{U^{2}}{C_{p}\left(T_{w}-T_{\infty}\right)}$ is the Eckert number.

Using Eq.(4.3), the boundary conditions (4.2) become

$$
\theta(0)=1 \quad \text { and } \quad \theta(\infty)=0 .
$$

The solution of Eq.(4.4) subject to the boundary conditions (4.5) is

$$
\theta(\eta)=e^{-S P_{r} \eta}+\frac{P_{r} E_{C}}{(S+2 \alpha)\left(S+2 \alpha-S P_{r}\right)}\left[a_{10} e^{-S P_{r} \eta}-\left(a_{10}+a_{11} \eta+a_{12} \eta^{2}\right) e^{-(S+2 \alpha) \eta}\right],
$$

for

$$
S P_{r} \neq S+2 \alpha
$$

and

$$
\theta(\eta)=e^{-S \operatorname{Pr} \eta}+\frac{\operatorname{PrEc}\left(a_{13} \eta+a_{14} \eta^{2}+a_{15} \eta^{3}\right) e^{-(S+2 \alpha) \eta}}{(S+2 \alpha)}, \text { for } \quad S \operatorname{Pr}=S+2 \alpha
$$

where

$$
a_{2}=\left(\frac{S}{2}+\alpha\right)^{2}+\beta^{2}+2 \sigma\left\{a_{1}\left(\frac{S}{2}+\alpha\right)+b_{1} \beta\right\}+\sigma^{2}\left(a_{1}^{2}+b_{1}^{2}\right)
$$




$$
\begin{aligned}
& a_{3}=2 \sigma\left\{a_{1}\left[\left(\frac{S}{2}+\alpha\right)^{2}+\beta^{2}\right]+\sigma\left(\frac{S}{2}+\alpha\right)\left(a_{1}^{2}+b_{1}^{2}\right)\right\} \\
& a_{4}=\sigma^{2}\left(a_{1}^{2}+b_{1}^{2}\right)\left\{\left(\frac{S}{2}+\alpha\right)^{2}+\beta^{2}\right\} \\
& a_{5}=\left(\frac{S}{2}+\alpha\right)\left\{\left(\frac{S}{2}+\alpha\right)^{2}+\beta^{2}\right\}+\sigma\left[a_{1}\left\{3\left(\frac{S}{2}+\alpha\right)^{2}+\beta^{2}\right\}\right]+ \\
& \left.+2 \beta b_{1}\left(\frac{S}{2}+\alpha\right)\right]+2 \sigma^{2}\left(\frac{S}{2}+\alpha\right)\left(a_{1}^{2}+b_{1}^{2}\right), \\
& a_{6}=2 \sigma a_{1}\left(\frac{S}{2}+\alpha\right)\left\{\left(\frac{S}{2}+\alpha\right)^{2}+\beta^{2}\right\}+\sigma\left(a_{1}^{2}+b_{1}^{2}\right)\left\{3\left(\frac{S}{2}+\alpha\right)^{2}+\beta^{2}\right\} \\
& a_{7}=\sigma^{2}\left(a_{1}^{2}+b_{1}^{2}\right)\left(\frac{S}{2}+\alpha\right)\left\{\left(\frac{S}{2}+\alpha\right)^{2}+\beta^{2}\right\}, \quad a_{8}=\frac{2(S+2 \alpha)-S \operatorname{Pr}}{(S+2 \alpha)(S+2 \alpha-S \operatorname{Pr})} \\
& a_{13}=a_{2}-\sigma S a_{5}-\frac{\left(a_{3}-\sigma S a_{6}\right)}{(S+2 \alpha)}+\frac{2\left(a_{4}-\sigma S a_{7}\right)}{(S+2 \alpha)^{2}}, \\
& a_{11}=2 a_{8}\left(a_{4}-\sigma S a_{7}\right)-\left(a_{3}-\sigma S a_{6}\right), \\
& a_{9}=\frac{(S+2 \alpha-S \operatorname{Pr})^{2}+(S+2 \alpha)(S+2 \alpha-S \operatorname{Pr})+(S+2 \alpha)^{2}}{(S+2 \alpha)^{2}(S+2 \alpha-S \operatorname{Pr})^{2}}
\end{aligned}
$$

Finally, we point out that no steady temperature distribution exists for blowing at the plate. From a physical point of view, this is due to the fact that the temperature at a given point continually increases due to conduction of heat away from the plate and convection of heat away from the plate due to blowing.

It is seen from Eqs (4.6) and (4.7) that the temperature distribution depends on the following dimensionless parameters (i) the elastic parameter (ii) the Prandtl number (iii) the Eckert number (which characterizes viscous dissipation in the flow) (iv) the suction parameter and (v) the rotation parameter.

Figure 6 shows the temperature distribution for various values of the elastic parameter $\sigma$ and rotation parameter $K^{2}$ with $\operatorname{Pr}=5.0, \mathrm{Ec}=2.0, S=2.0$. It is observed that the temperature at a point increases with an increase in either $\sigma$ or $K^{2}$. The variations of temperature distribution $\theta$ for different values of the Eckert number Ec and suction parameter $S$ are shown in Fig.7. It is found that the temperature 
at a point increases with an increase in the Eckert number whereas it decreases with an increase in the suction parameter.

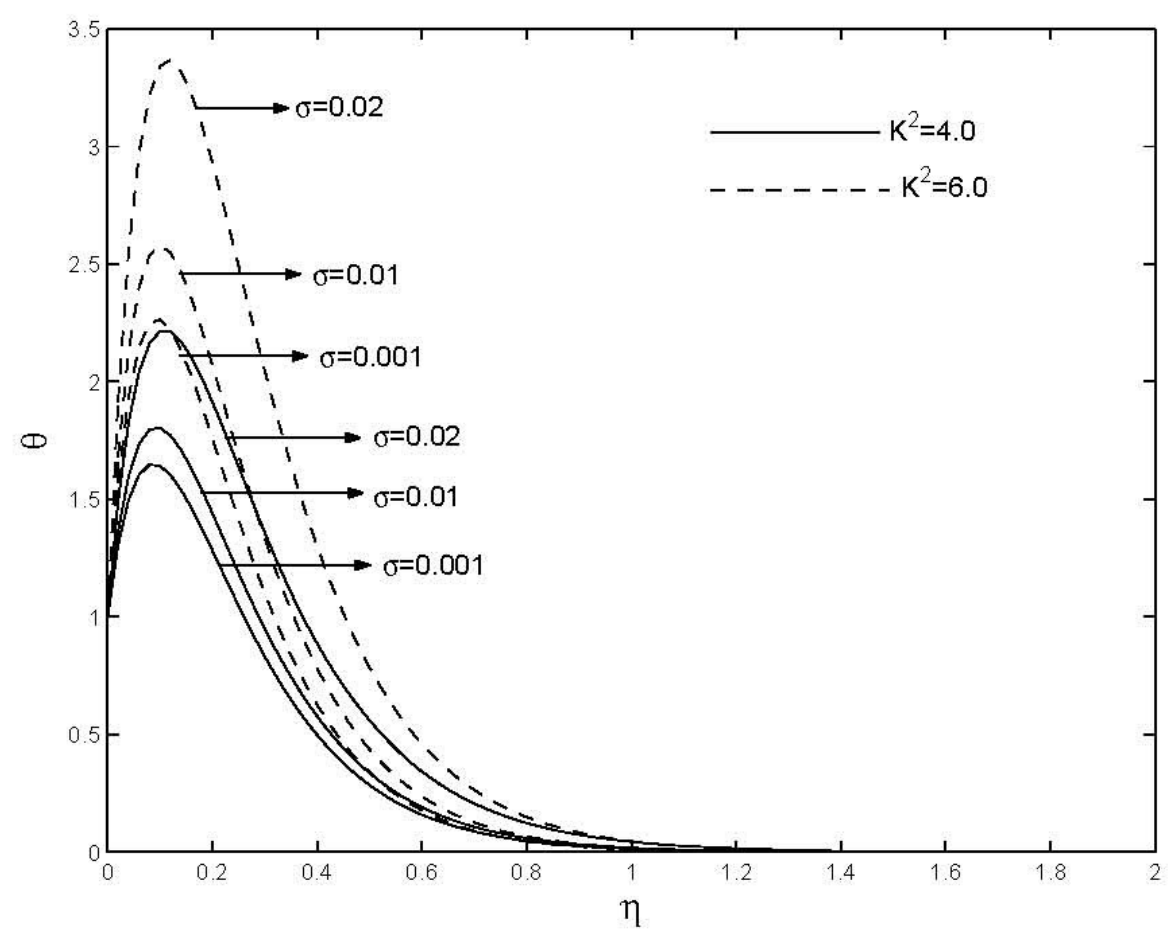

Fig.6. Variations of temperature profile $\theta$ for $\operatorname{Pr}=5.0, S=2.0, \mathrm{Ec}=2.0$.

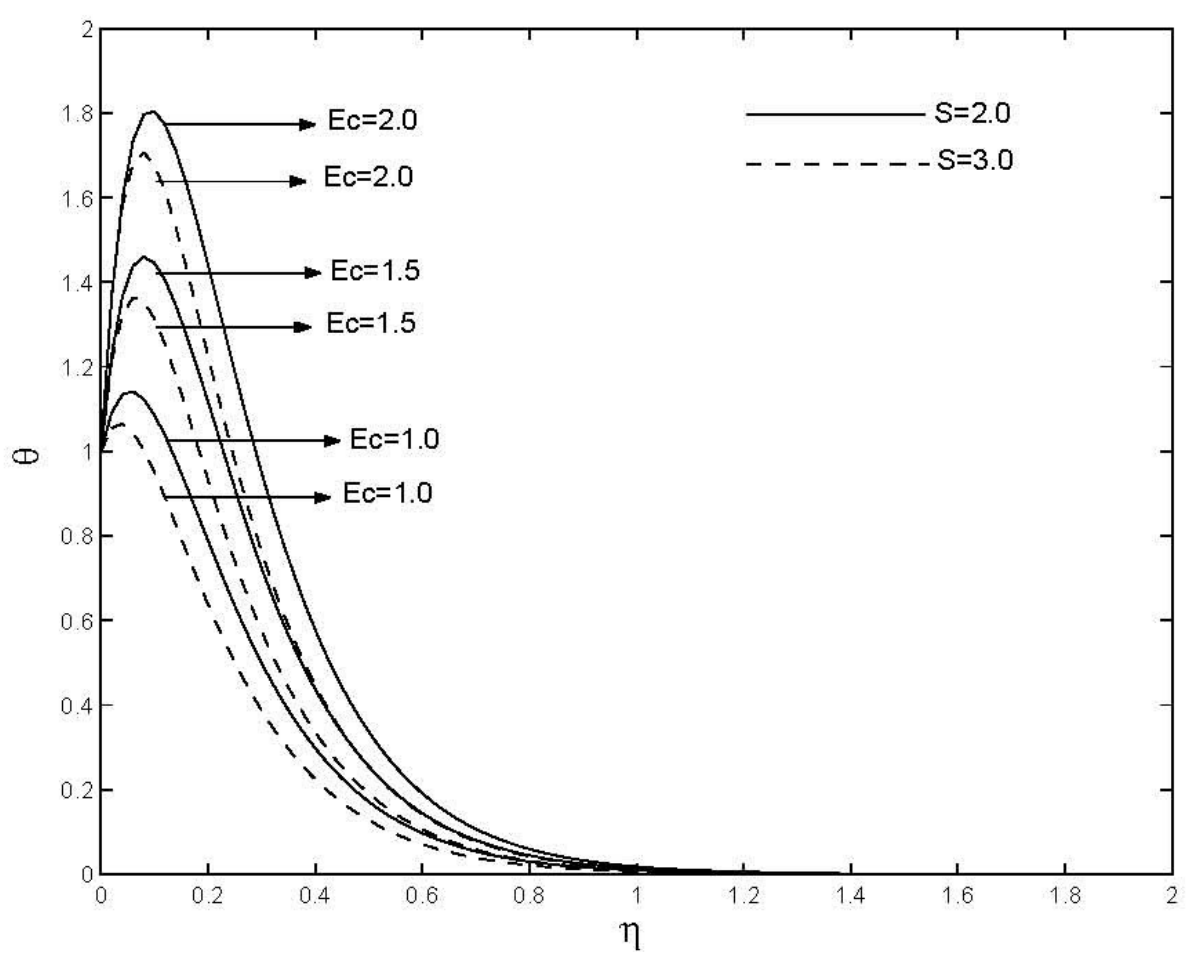

Fig.7. Variations of temperature profile $\theta$ for $\operatorname{Pr}=5.0, \sigma=0.01, K^{2}=4.0$. 
The non-dimensional rate of heat transfer at the plate $\eta=0$ is given by

$$
\begin{aligned}
& q_{w}=\left(\frac{d \theta}{d \eta}\right)_{\eta=0}, \\
& =-S \operatorname{Pr}+\frac{\operatorname{PrEc}\left[(S+2 \alpha-S \operatorname{Pr}) a_{10}-a_{11}\right]}{(S+2 \alpha)(S+2 \alpha-S \operatorname{Pr})}, \quad \text { for } \quad S+2 \alpha \neq S \operatorname{Pr},
\end{aligned}
$$

and

$$
q_{w}=-S \operatorname{Pr}+\frac{\operatorname{PrEc} a_{13}}{(S+2 \alpha)}, \quad \text { for } \quad S+2 \alpha=S P .
$$

The values of $q_{w}$ are given in Tab.3 for various values of $\sigma$ and $K^{2}$ with Ec=1.0, $S=2.0$ and $\operatorname{Pr}=5.0$. It is seen that the rate of heat transfer increases with an increase in either the elastic

\begin{tabular}{|c|c|c|c|c|}
\hline & & $q_{w}$ & & \\
\hline$K^{2} \sigma$ & 0.001 & 0.01 & 0.03 & 0.05 \\
\hline 4.0 & 4.31434 & 5.99896 & 9.54853 & 14.77307 \\
\hline 6.0 & 12.26702 & 15.49967 & 15.24312 & 22.42388 \\
\hline 8.0 & 24.09315 & 29.69641 & 40.45284 & 55.74079 \\
\hline 10.0 & 44.19298 & 53.67467 & 70.63224 & 94.01682 \\
\hline
\end{tabular}
parameter $\sigma$ or rotation parameter $K^{2}$.

Table.3. Rate of heat transfer $q_{w}$ for $\mathrm{Ec}=1.0, \operatorname{Pr}=5.0, S=2.0$

It follows from Eqs (4.9) and (4.10) that when $\mathrm{Ec}=E_{\text {crit }}$ where

$$
E_{\text {crit }}=\frac{S(S+2 \alpha)(S+2 \alpha-S \operatorname{Pr})}{(S+2 \alpha-S \operatorname{Pr}) a_{10}-a_{11}}, \quad \text { for } \quad S+2 \alpha \neq S \operatorname{Pr}
$$

and

$$
E_{c r i t}=\frac{(S+2 \alpha)}{a_{13}} \quad \text { for } \quad S+2 \alpha=S \operatorname{Pr}
$$

then there is no flow of heat either from the plate to the fluid or from the fluid to the plate. We calculated the critical Eckert number $E_{\text {crit }}$ for different values of $\sigma$ and $K^{2}$ which are given in Tab.4. 
Table.4. Critical Eckert number for $S=2.0, \operatorname{Pr}=5.0$.

\begin{tabular}{|l|llll|}
\hline & \multicolumn{5}{|c|}{$E_{\text {crit }}$} \\
\hline$K^{2} \sigma$ & 0.001 & 0.01 & 0.02 & 0.03 \\
\hline 4.0 & 0.69860 & 0.62504 & 0.51154 & 0.40366 \\
6.0 & 0.44909 & 0.39216 & 0.31214 & 0.24086 \\
8.0 & 0.29331 & 0.25191 & 0.19820 & 0.15211 \\
10.0 & 0.18452 & 0.15704 & 0.12401 & 0.09613 \\
\hline
\end{tabular}

It follows from Eqs (4.9) and (4.11) that heat will flow from the plate to the fluid if $\mathrm{Ec}<E_{\text {crit }}$, while heat will flow from the fluid to the plate if $\mathrm{Ec}>E_{c r i t}$. It is found that the critical Eckert number decreases with an increase in the elastic parameter $\sigma$. It is also found that $E_{c r i t}$ is always smaller in the presence of rotation than without rotation. Hence, heat will flow from the fluid to the plate at a smaller value of the Eckert number than the corresponding value of it in the absence of rotation.

\section{Nomenclature}

$$
\begin{aligned}
& \mathrm{E}_{\mathrm{c}}\left(=\frac{U^{2}}{c_{p}\left(T_{w}-T_{\infty}\right)}\right) \text { - Eckert number } \\
& E_{\text {crit }}-\text { critical Eckert number } \\
& K^{2}\left(=2 \Omega v / U^{2}\right) \text { - rotation parameter } \\
& k_{0} \quad \text { - short memory coefficient } \\
& N(\tau) \text { - distribution function of relaxation time } \tau \\
& \operatorname{Pr}\left(=\mu c_{p} / \lambda\right) \quad-\text { Prandtl number } \\
& q_{w} \text { - rate of heat transfer } \\
& S\left(=v_{0} / U\right) \text { - suction parameter } \\
& T \text { - fluid temperature } \\
& T_{w} \text { - plate temperature } \\
& T_{\infty} \quad \text { - temperature at infinity } \\
& t^{\prime}-\text { time } \\
& u, v, w \text { - velocity components in } x, y \text { and } z \text { directions respectively } \\
& u_{1}, w_{l}(=u / U, w / U) \text { - non-dimensional velocity components } \\
& v_{0}-\text { suction velocity } \\
& \alpha, \beta, \alpha_{1}, \beta_{1}-\text { are defined in Eq.(2.28) } \\
& \eta(=U y / v) \text { - non-dimensional normal coordinate axis } \\
& \eta_{0} \quad \text { - limiting viscosity at small rate of shear } \\
& \theta\left(=\frac{T-T_{\infty}}{T_{w}-T_{\infty}}\right)-\text { non-dimensional temperature } \\
& \lambda-\text { thermal diffusivity } \\
& \mu-\text { coefficient of viscosity } \\
& v \text { - kinematic viscosity }
\end{aligned}
$$




$$
\begin{aligned}
\sigma & - \text { fluid density } \\
\sigma\left(=\frac{k_{0} U^{2}}{\rho v^{2}}\right) & - \text { visco-elastic parameter } \\
\Omega & - \text { angular velocity }
\end{aligned}
$$

\section{References}

Beard D.W. and Walters K. (1964): Elastico-viscous boundary layer flows. - Proc. Cambridge Philos. Soc. Maths. Phys. Sci., vol.60, pp.667-674.

Joseph D.D. (1990): Fluid Dynamics of Visco-Elastic Liquids. - New York: Springer-Verlag.

Kaloni P.N. (1966): Fluctuating flow of an elastico-viscous fluid past a porous flat plate. - Physics of Fluids, vol.10, pp.1344-1346.

Rajagopal K.R. and Gupta A.S. (1984): An exact solution for the flow of a non-Newtonian fluid past an infinite porous plate. - Meccanica, vol.19, pp.158-160.

Ray Mahapatra T. and Gupta A.S. (2004): Stagnation-point flow of a viscoelastic fluid towards a stretching surface. Int. J. of Non-Linear Mech., vol.39, pp.811-820.

Received: November 12, 2011

Revised: July 3. 2012 